Meta

Journal des tradlucteurs

Translators' Journal

\title{
La liberté en traduction
}

\section{Karla Déjean Le Féal}

Volume 36, numéro 2-3, juin 1991

URI : https://id.erudit.org/iderudit/003449ar

DOI : https://doi.org/10.7202/003449ar

Aller au sommaire du numéro

Éditeur(s)

Les Presses de l'Université de Montréal

ISSN

0026-0452 (imprimé)

1492-1421 (numérique)

Découvrir la revue

Citer cet article

Déjean Le Féal, K. (1991). La liberté en traduction. Meta, 36(2-3), 448-455.

https://doi.org/10.7202/003449ar

Ce document est protégé par la loi sur le droit d'auteur. L'utilisation des services d'Érudit (y compris la reproduction) est assujettie à sa politique d'utilisation que vous pouvez consulter en ligne.

https://apropos.erudit.org/fr/usagers/politique-dutilisation/
Cet article est diffusé et préservé par Érudit.

Érudit est un consortium interuniversitaire sans but lucratif composé de l'Université de Montréal, l'Université Laval et l'Université du Québec à Montréal. Il a pour mission la promotion et la valorisation de la recherche. https://www.erudit.org/fr/ 


\section{LA LIBERTÉ EN TRADUCTION}

KARLA DÉJEAN LE FÉAL

Université de Paris III, Paris, France

La liberté en traduction, voilà un thème qui ne laisse indifférents ni les traducteurs en herbe ni les traducteurs confirmés; sinon, pourquoi tant de débats, de controverses, ou de colloques?

Pourtant, on a quelques difficultés à voir l'objet de toute cette agitation, si l'on fait abstraction de la traduction littéraire, sur laquelle il ne m'appartient pas de me prononcer faute d'expérience pratique. En effet, il y a lieu de se demander si, pour la traduction fonctionnelle, cette liberté dont on parle tant n'est pas plutôt un mythe qu'une réalité.

Selon la théorie moderne de la traduction, cette dernière consiste à dégager du texte source le vouloir dire de son auteur, puis de réexprimer ce vouloir dire dans la langue cible, et ce dans le même registre de langue, avec le même soin rédactionnel et pour des lecteurs de la même catégorie socio-professionnelle, bien qu'imprégnés d'une culture différente.

Cela dit, voyons dans quelle mesure cette définition permet au traducteur d'exercer une quelconque liberté. Pour répondre à cette question, il faut passer en revue successivement les deux phases du processus de traduction, en commençant par la première, celle de l'analyse du texte.

\section{LA LIBERTÉ AU STADE DE L'ANALYSE}

Étant donné que le traducteur travaille normalement à partir d'un texte fini, le vouloir dire de l'auteur doit être considéré comme donné. La tâche du traducteur consistera donc à dégager ce vouloir dire, sa partie explicite ainsi que sa partie implicite. Il y parviendra grâce

- à ses connaissances de la langue source qui lui permettent d'accéder au contenu cognitif proprement dit du texte et de discerner les caractéristiques de la forme qui déterminent le registre et, donc, la perspective choisie par l'auteur pour traiter son sujet;

- à la mobilisation de ses connaissances du sujet traité, mobilisation nécessaire à la reconstitution du raisonnement de l'auteur dans le but d'appréhender la totalité de l'implicite contenu dans le texte;

- à ses informations sur la qualité en laquelle l'auteur s'exprime et sur le but qu'il poursuit, ce qui met le traducteur en mesure de situer le texte par rapport à la réalité et d'en apprécier l'enjeu.

Le traducteur s'assure de l'exactitude de son analyse par l'épreuve de cohérence du sens dégagé, et ce au niveau du texte tout entier. Il vérifie en outre qu'il saisit à tout moment non seulement ce que l'auteur a voulu exprimer, mais encore où celui-ci voulait en venir en l'exprimant.

$\mathrm{Au}$ cas où, lors de ses vérifications, le traducteur constaterait une quelconque incohérence, il ne pourrait évidemment pas prendre la liberté de l'ignorer ni de l'éliminer en substituant au vouloir dire de l'auteur un raisonnement qui lui semblerait plus cohérent. De même, s'il ne voyait pas le rapport entre telle observation et telle autre, il ne pourrait pas prendre la liberté d'en faire abstraction. Bien au contraire, ce type d'incohérence ou 
de perplexité doit l'inciter à approfondir son analyse, à compléter ses informations jusqu'à ce que l'incohérence perçue se résorbe d'elle-même ou que le lien entre les énoncés ou parties d'énoncés qui lui avaient semblé décousus apparaisse clairement.

Rien que des contraintes, donc, et aucune liberté pour le traducteur dans la phase d'analyse du processus.

\section{LA. LIBERTÉ AU STADE DE LA RÉEXPRESSION}

Que se passe-t-il alors au stade de la reformulation du sens dégagé? Le traducteur se doit de réexprimer ce sens dans le même esprit que l'auteur, dans le même registre, avec le même soin rédactionnel et le même souci de communication avec ses lecteurs. Ce dernier impératif peut l'amener, le cas échéant, à expliciter ce qui était implicite dans le texte original, ou à rendre implicite ce qui était explicite, pour tenir compte d'éventuelles différences entre les connaissances préexistantes des lecteurs de la langue source et des lecteurs de la langue cible.

Il jouit, à l'intérieur de ces limites, de la liberté de choisir les mots que lui laisse la langue cible ainsi que les règles d'emploi, écrites ou non, de cette langue et du technolecte concerné. Et encore la nécessité de rebâtir non pas une série de phrases, mais un texte intelligible et lisible vient-elle réduire plus ou moins fortement l'éventail de son choix. C'est particulièrement vrai dans les cas où la langue cible ne tolère pas la répétition de termes, non techniques, à peu de distance les uns des autres.

Il n'en reste pas moins que le traducteur garde une certaine latitude quant au choix des expressions, comme le montrent ces quatre traductions faites par quatre personnes différentes ${ }^{1}$ :

\section{Congé du Nouvel An}

Monsieur Kim, sa fermme et sa fille, partis dans une station thermale pour leur congé de Nouvel An, ont finalement dû rentrer chez eux le jour même de leur départ pour arriver à 2 heures le lendemain matin après une journée harassante.

Arrivés à Onyang, dans la région de Chungchungramdo, à 4 heures de l'aprèsmidi, ils pensaient trouver au moins une chambre libre dans cette station qui compte tant d' hôtels. C'était un mauvais calcul. Tout était complet. Monsieur Kim et sa famille allèrent alors à Dogo, puis à Ducksan, les stations thermales les plus proches, en regrettant de ne pas avoir téléphoné avant leur départ.

Les Coréens se comportent de manière similaire en se rendant à l'hôpital sans prendre rendez-vous. Il en résulte que pour une consultation de quelques minutes, ils attendent plusieurs heures, ce qu'ils considèrent comme normal. À l'étranger, avant d'aller à une consultation à l' hôpital, on fixe par téléphone une date et une heure précises, ce qui évite de perdre du temps.

Au Japon, téléphoner pour réserver une chambre relève du simple bon sens, même quand il s'agit d' une petite auberge en province.

\section{Le prix de l'imprévoyance}

Monsieur Kim, parti pour passer le nouvel an à Onyang, station thermale dans la région de Chungchungramdo, accompagné de sa femme et de sa fille, ne devait finalement faire qu'un aller et retour. Il rentra bredouille en plein milieu de la nuit, plutôt mécontent de son expédition.

Comme il était persuadé qu' il trouverait sans problème une chambre dans un des nombreux hôtels de la place, il n'avait pas fait de réservation. Il s'en mordit les doigts quand, arrivé à destination au milieu de l'après-midi, il constata que tous les hôtels affichaient complet non seulement à Onyang, mais aussi à Dojo et Ducksan, deux stations voisines. 
Les Coréens ont la fâcheuse habitude de ne pas s'annoncer à l'avance non seulement en voyage, mais aussi lorsqu'ils vont consulter à l' hôpital. Et il n'est pas rare qu'ils y passent alors plusieurs heures pour un examen de quelques minutes.

Il serait pourtant si facile d'éviter ce gaspillage de temps en prenant rendez-vous à l'avance, comme cela se pratique couramment à l'étranger. Au Japon, par exemple, personne n'aurait l'idée de se rendre dans la plus petite auberge de province sans réservation.

\section{Congé de Nouvel An}

Parti avec sa femme et sa fille pour la station thermale d'Onyang dans la région du Chungchungramdo, avec l'intention d'y passer son congé de Nouvel An, Monsieur Kim s'est vu contraint de regagner ses pénates à 2 heures du matin, après une journée exténuante.

Ils étaient arrivés à Onyang à 4 heures de l'après-midi, convaincus que, vu le nombre des hôtels, ils trouveraient bien une chambre de libre. Mauvais calcul: tous les hôtels affichaient complet.

Ils se rendirent alors aux stations voisines de Dogo et de Ducksan. En vain.

C'est alors que Monsieur Kim commença à regretter sérieusement de n'avoir pas fait de réservation...

On retrouve la même tendance des Coréens à se déplacer sans rendez-vous, quand il s'agit d'aller en consultation à l'hôpital. C'est ce qui explique qu'un examen de quelques minutes seulement se paie, la plupart du temps, de plusieurs heures d'attente.

Nous gaspillons ainsi un temps précieux, contrairement aux étrangers, qui ont l' habitude d'appeler l' hôpital pour fixer la date et l' heure de la consultation.

Au Japon, par exemple, la pratique de la réservation est courante, même s'il ne s'agit que de la plus modeste des auberges de province.

\section{Les vacances avortées de M. Kim}

Monsieur Kim s'était rendu, avec sa femme et sa fille, dans une station thermale pour y passer son congé de nouvel an. Il était déjà de retour le lendemain à 2 heures du matin complètement épuisé par une journée de déceptions.

La petite famille était arrivée à Onyang, une ville de la province de Chungchungramdo, la veille à 16 heures et pensait trouver sans aucun problème au moins une chambre d'hôtel libre dans une ville comme Onyang, où les possibilités d'hébergement ne manquent pas. Mais comme il ne faut pas vendre la peau de l'ours avant de l'avoir tué...

Comme ils ne réussirent pas à trouver la moindre chambre libre, ils partirent tenter leur chance à Dogo, puis à Ducksan, deux stations thermales des environs. En vain.

Et notre $M$. Kim de regretter son imprudence: "Si seulement j’avais téléphoné avant notre départ pour réserver une chambre!».

Nombreux sont les Coréens qui agissent avec le manque de prévoyance de M. Kim; lorsqu'ils doivent aller à l' hôpital par exemple. Ils acceptent comme une chose tout à fait normale d'attendre de longues heures pour une consultation qui ne dure que quelques minutes.

C'est ainsi que nous gaspillons bêtement un temps précieux par rapport à d'autres pays où les gens ont le réflexe de fixer par téléphone le jour et l' heure de leur consultation. Au Japon, par exemple, le fait de réserver relève du bon sens le plus élémentaire, même s'il s'agit d' une petite auberge perdue dans la province.

On voit que ces quatre traductions contiennent la même information présentée dans le même registre. Elles ont été jugées conformes à l'original sous l'angle de ces deux critères et au moins équivalentes à celui-ci sous l'angle de la qualité rédactionnelle. 
Voici le texte d'origine:

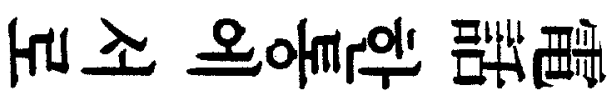

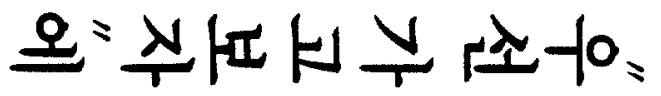

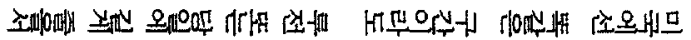

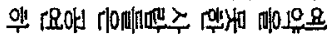

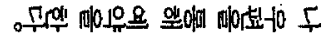

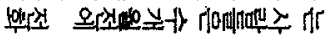

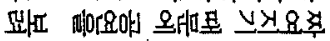

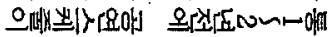

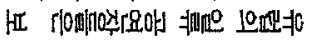

\section{오바 개꼬유요밤저}

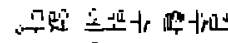

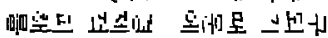

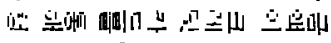

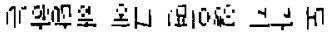

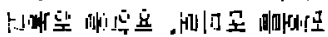

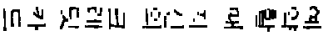

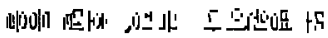
,

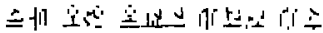
게

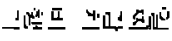

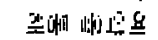

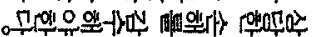

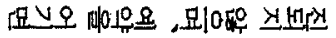

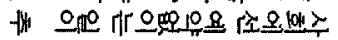

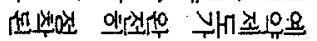
.

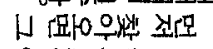

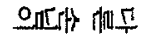

104.

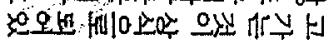

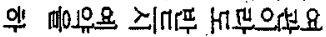

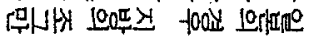

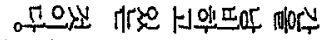

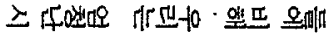

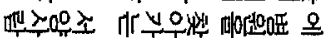

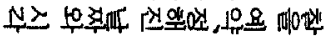

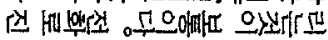

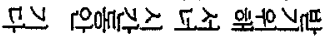

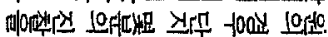

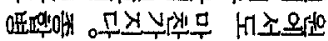

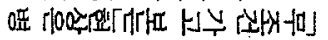

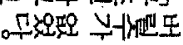

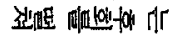

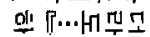

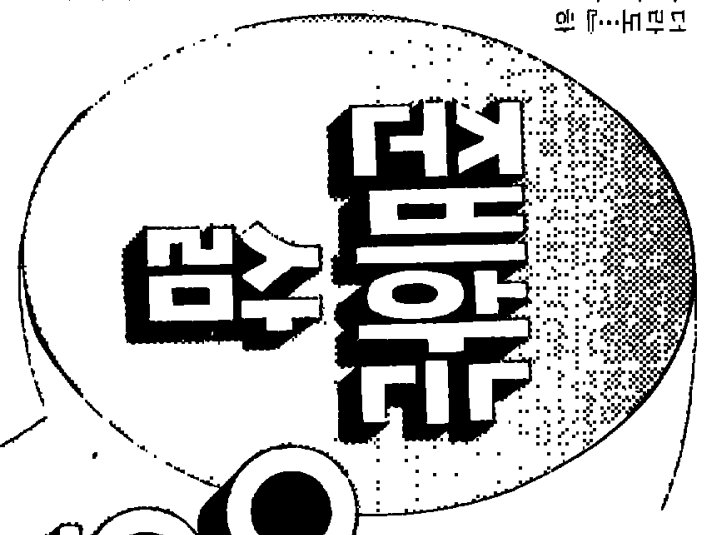

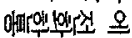

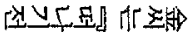

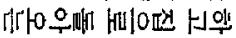

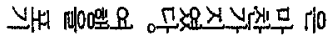

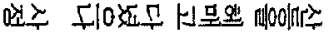
近, 水

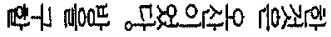

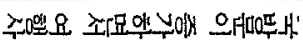

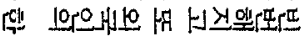

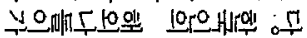

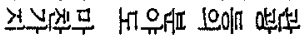

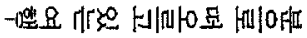

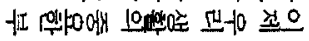

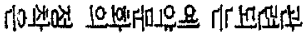
$\forall \forall 0 \geq 0$ 에 $\quad$ 서

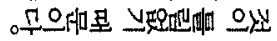

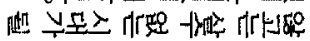

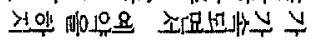

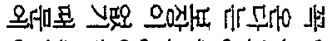

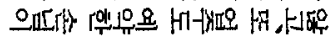

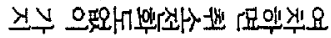

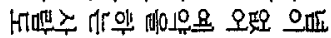

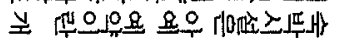

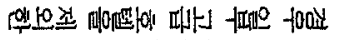

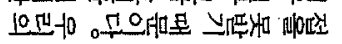

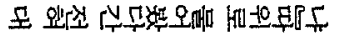

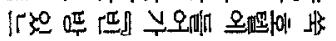

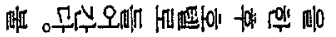

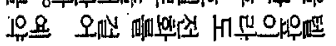

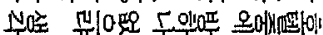

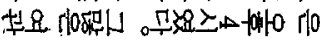

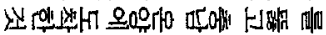

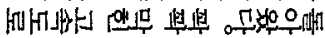

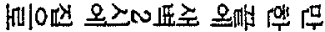

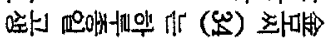

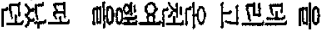

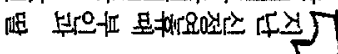
토우 으옹 뜨태저곤 
Pourtant, les mots et les expressions employés dans ces traductions ne sont pas identiques. Le traducteur jouit donc plus ou moins de la même liberté que l'auteur qui est arrivé au bout du processus de conception de l'idée, au stade où il ne reste plus qu'à mouler celle-ci dans une forme linguistique appropriée en tenant compte du registre choisi et du public visé.

Par conséquent, la liberté dont dispose le traducteur n'est pas une liberté en traduction à proprement parler, mais une liberté purement rédactionnelle.

Les quatre traductions présentées plus haut illustrent bien cela puisqu'elles ont été réalisées selon des modalités un peu différentes de celles qui sont la règle en traduction manuelle. Ces modalités ont été décrites en détail dans The Interpreters Newsletter $\mathrm{n}^{\circ} 2$, de l'Université de Trieste sous le titre «Interpretation's contribution to machine translation». Ce qui nous intéresse ici c'est le fait que ces quatre versions en français sont issues d'un travail de pure rédaction. Ceux qui l'ont effectué ne connaissaient d'ailleurs pas la langue source. De plus, deux d'entre eux n'étaient pas des traducteurs. Leur tâche consisait à rebâtir un texte en français sur la base d'un condensé transcodé du contenu cognitif du texte source et de consignes stylistiques présentés sous la forme suivante:

\section{Explication des symboles utilisés}

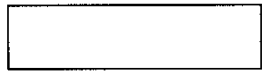

$(+)$

... $/ \ldots$

$\because$

$/ \mathrm{t}$

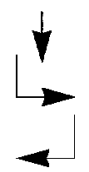

sujet de la phrase

pluriel

significations au choix

raison

contrairement

pour, pour que

proposition subordonnée

passé

Notes transcodées à partir d'un texte écrit en coréen paru dans un quotidien grand public; style journalistique. 


\section{Congé du nouvel an}
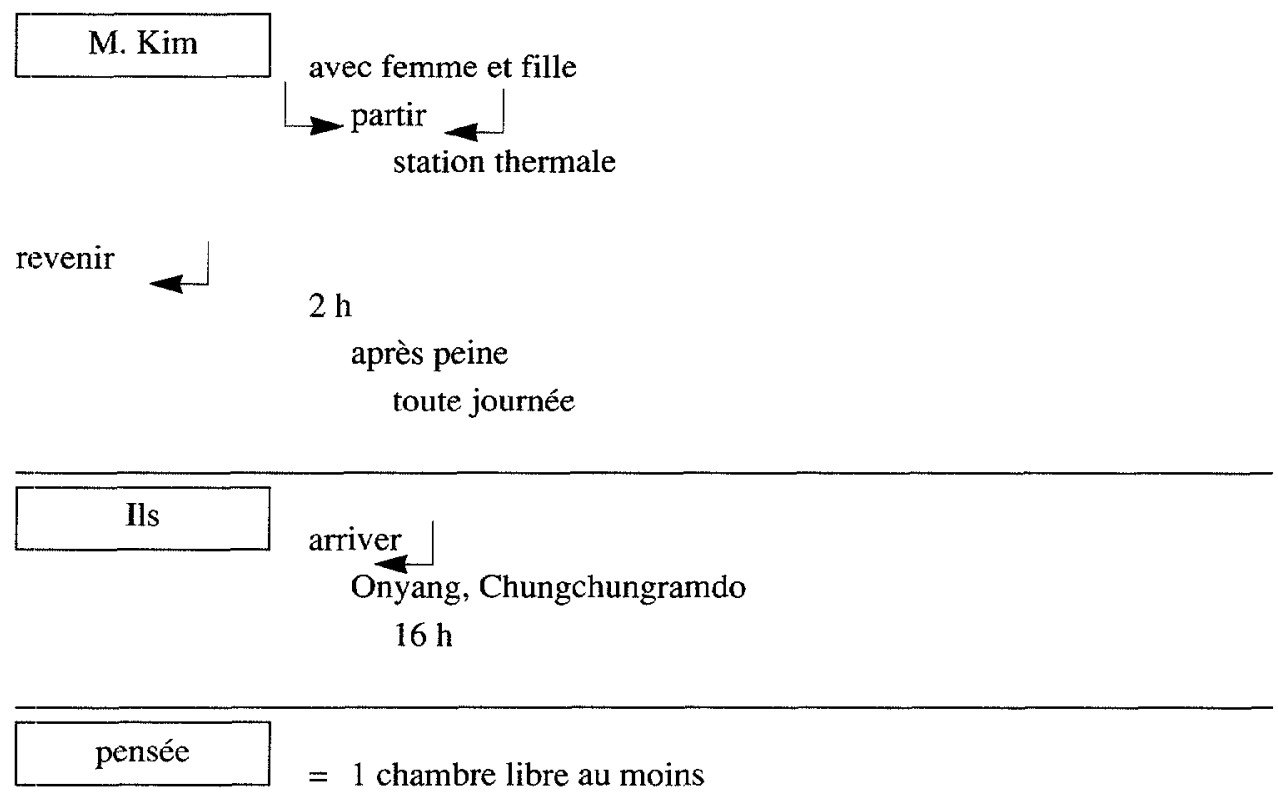

$=1$ chambre libre au moins dans tant hôtel (+)

être

faux/mauvais calcul

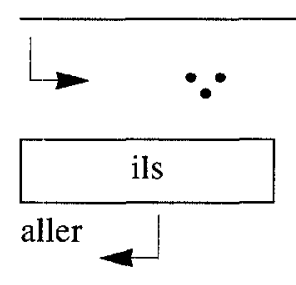

pas chambre libre

Dogo et Ducksan

$=$ station $(+)$ thermale proche

M. Kim

regretter

pas téléphoner

avant départ 


\section{Phénomène}

$=$ se rendre sur place tout de suite

s'appliquer

hôpital (+)

Hôpital (+) $\downarrow$

consultation quelques minutes (+)

attendre plusieurs heures

être normal

réservation téléphonique

consultation

/t étranger (+)

aller hôpital

$\longrightarrow \quad$ heure, date fixées

nous

gaspiller

beaucoup heures

Japon

même auberge petite

province

réservation

être sens commun/bon sens/banal

Ce condensé et ces consignes de style peuvent être considérés comme une matérialisation - imparfaite, certes, mais pas forcément insuffisante pour autant - de ce que le traducteur a «en tête» au terme de son analyse, donc au moment où il s'apprête à reformuler le vouloir de l'auteur. Nous avons vu plus haut que ce vouloir dire ainsi que le registre choisi en fonction du public visé, constituent, pour le traducteur, des données immuables; seule la manière dont il rend à ces données leur matérialité dans la langue cible est laissée à son appréciation. Et comme l'a fait l'auteur avant lui, après avoir décidé ce qu'il voulait exprimer et quelle perspective il voulait adopter, le traducteur choisit la forme linguistique selon son style personnel et son sens esthétique de la langue. 
Or ce style et ce sens sont également des quasi-données pour chacun de nous, certes susceptibles d'évoluer, mais relativement peu élastiques à chaque instant précis de notre existence. En d'autres termes, le traducteur, comme l'auteur, est plus ou moins prisonnier de son idiolecte au moment où il rédige. Vue sous cet angle, même l'apparente liberté rédactionnelle du traducteur perd beaucoup de sa substance.

Elle se réduit en fait au droit du traducteur de rester lui-même dans l'exercice de son métier si, toutefois, ses compétences en matière de rédaction sont au moins égales à celles des auteurs qu'il traduit. Et comme il n'est de toute façon guère convenable qu'on puisse faire abstraction de sa personnalité dans ses activités intellectuelles, ce droit doit être considéré comme une condition sine qua non de toute traduction. En effet, le contester signifierait contester la légitimité même de l'activité traduisante. Ce serait d'ailleurs difficile, faute de critères objectifs, puisque toute discrimination entre idiolectes de qualité égale serait forcément subjective. Il est cependant tout aussi difficile de considérer ce droit comme une liberté au sens habituel du terme.

En fin de compte, la seule vraie liberté qui subsiste pour le traducteur est celle d'avoir, dans la langue cible, un style personnel plus fluide et un sens esthétique plus fin que l'auteur dans la langue source, et de rédiger son texte avec plus de soin. Et même cette liberté n'est réelle que dans le cas où l'auteur s'est exprimé dans sa langue maternelle. Dans le cas contraire, le traducteur se doit de lui être supérieur dans l'art de manier la langue.

En conclusion, si l'on examine la liberté en traduction fonctionnelle à la lumière de la théorie moderne de la traduction, on constate que cette liberté n'existe qu'au stade de la réexpression du vouloir dire de l'auteur, et seulement sous la forme de la latitude laissée, dans cette phrase, au traducteur de surpasser l'auteur en excellence... S'il en est capable.

La seule question qu'on pourrait donc poser serait celle de savoir si le traducteur peut prendre la liberté de mieux rédiger dans sa langue que l'auteur dans la sienne et de le faire avec plus de soin. Mais cette question vaut-elle vraiment la peine d'être posée?

\section{NOTE}

1. Qu'elles soient remerciées ici de leur concours. 